\title{
Patterning and electrical interfacing of individually controllable conducting polymer microactuators
}

\author{
Edwin Jager, Nirul Masurkar, Nnamdi Felix Nworah, Babita Gaihre, Gursel Alici and \\ Geoffrey M. Spinks
}

\section{Linköping University Post Print}

\section{Tweet}

N.B.: When citing this work, cite the original article.

Original Publication:

Edwin Jager, Nirul Masurkar, Nnamdi Felix Nworah, Babita Gaihre, Gursel Alici and Geoffrey M. Spinks, Patterning and electrical interfacing of individually controllable conducting polymer microactuators, 2013, Sensors and actuators. B, Chemical, (183), 283289.

http://dx.doi.org/10.1016/j.snb.2013.02.075

Copyright: Elsevier http://www.elsevier.com/

Postprint available at: Linköping University Electronic Press http://urn.kb.se/resolve?urn=urn:nbn:se:liu:diva-96174 


\title{
Patterning and electrical interfacing of individually controllable conducting polymer microactuators
}

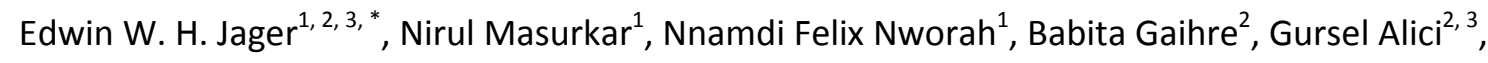
Geoffrey M. Spinks, ${ }^{2,3}$

1. Linköping University, Department of Physics, Chemistry and Biology, Biosensors and Bioelectronics Centre, 58183 Linköping, Sweden

2. ARC Centre of Excellence in Electromaterials Science, Intelligent Polymer Research Institute, University of Wollongong, Wollongong, NSW, 2522, Australia.

3. School of Mechanical, Materials and Mechatronic Engineering, University of Wollongong, Wollongong, NSW, 2522, Australia.

* Corresponding Author:

edwin.jager@liu.se; +46-13-281246

Sensors \& Actuators: B

\begin{abstract}
Conducting polymer actuators such as polypyrrole (PPy) microactuators are interesting candidates to drive autonomous microrobotic devices that require low weight and low power. Simple PPy tri-layer bending type microactuators that operate in air have been demonstrated previously but they lack individual control and had problems with short circuiting due to electrical connections. The lack of micropatterning methods and proper interfacing are currently major obstacles in the development of PPy tri-layer microactuators. Here, we report for the first time methods for successfully patterning and interfacing of such tri-layer PPy microactuators. The PPy tri-layer actuators were patterned using adapted microfabrication technology including photolithography. The interface was based on a flexible printed circuit board comprising the electronic circuit into which the actuator unit was embedded. It showed that the microfabricated tri-layer actuators functioned as good as the normally fabricated actuators. The new interface seemed to actually improve the actuator performance. This interfacing method could also be applied to other electroactive polymer devices, such as ion polymer metal composites (IPMC) and dielectric elastomers (DE).
\end{abstract}

\section{Keywords}

Polypyrrole; actuator; microfabrication; electrical interface; Electroactive polymer actuators; PVDF membrane 


\section{Introduction}

One of the obstacles in front of establishing functional microrobotic devices is the absence of small footprint actuators, which are not only compliant, but also need minimum electric power so that they can operate (battery powered) autonomously and comply with various operation environments. Although significant research efforts have been dedicated to traditional macro-sized robotic systems, the progress in developing microrobotics has been very limited due to the difficulty of powering and actuating them using onsite means such as an electric motor and a battery with enough energy density. Existing actuation and power supplies cannot be scaled further to realize functional robotic systems. Actuators based on electromechanically active polymers (EAP) and especially conducting polymers (CP) are very suitable for establishing functional autonomous micro-robotic systems, since they are light-weight, are operated at low potentials (1-2 V), move silently and can be fabricated at the microscale [1]. However, their limited operational life, limited structural strength to support a useful payload, and the need for robust, compact and low resistance electrical contacts still remain as challenges.

These conductive polymers such as polypyrrole (PPy) can be electrochemically oxidized and reduced. This reversible redox reaction is accompanied by a volume change of the material caused by the ingress and egress of ions and solvent from the electrolyte. This volume change has been used to build polymer actuators [2-5]. The technology has been down scaled to produce PPy-microactuators $[1,6]$. These PPy-microactuators are traditionally formed as PPy/Au bending bilayers, although even microactuator using the bulk expansion have been demonstrated $[7,8]$. The PPy-Au bilayer microactuators have been employed as hinges lifting Si-plates [6,9] and as joints in a complex microrobot [10]. The PPy (micro-)actuators are commonly operated in an aqueous, external electrolyte. To allow for operation in air, actuator configurations have been developed where the electrolyte (non-aqueous or an ionic liquid) is embedded. These systems are either based on an interpenetrating network of a CP with a structural layer [11] or a multilayer assembly comprising two CP layers on both side of an electrolyte-containing membrane [12]. Recently, both systems have been down scaled to the microscale $[13,14]$. However, these are still simple, individual bending beam like microactuators. Complex microdevices such as microrobotics would comprise multiple and as well as articulated CP microactuators. However assembling single actuators in the microdevice is cumbersome at this small scale. Preferably the actuator unit is fabricated as a monolithic unit comprising individual addressable actuators. This requires novel methods to pattern and microfabricate $\mathrm{CP}$ actuators. Kruusamäe et al have patterned the electrode surface of Ionic Polymer Metal Composite actuators by scratching off the conducting material using a sharp metal blade [15]. However this method does not provide the resolution needed for microscale actuators nor does it allow batch fabrication, which is the aim of our work.

Electrical connections to EAP devices and especially CP actuators have always been a cumbersome step in the interface of such devices. Alligator clips, Kelvin clips (alligator clips with separately addressable sides), or home made tweezers have been used. These systems all use mechanical pressure to form a satisfactory junction between the conducting polymer and connecting wires, but all are large in size and the mechanical pressure can sometimes damage the CP as well as cause short circuiting. For $\mathrm{CP}$ microactuators and microsystems comprising $\mathrm{CP}$ microactuators these issues are more important since the size of the interfacing system must be minimized and the microactuators are relatively fragile. For these systems, more elaborate connections are required. Although probe 
needles and wire bonding have been used, these methods are not very stable nor successful. We are currently developing a range of novel microdevices based on individual addressable CP tri-layer microactuators that work in air [16] and patterning and the method of interfacing are currently a major obstacles in their development. Here, we report for the first time methods for successfully patterning and interfacing of such tri-layer microactuators.

The primary contribution of this study is to develop methods to pattern and to form stable electrical connections for bending type CP microactuators that can be individually controlled. The interfacing system requirements include a low ohmic contact between the $\mathrm{CP}$ and a noble metal (or carbon) connecting wire; a mechanically robust connection that can allow CP bending operation; and minimum size.

\section{PPy tri-layer microactuators}

The structure of the tri-layer actuators considered in this study is illustrated in Fig. S1, in which the two outer PPy layers are the active components as the electrodes and an inner porous separator of poly(vinylidene fluoride) (PVDF) holds the liquid electrolyte consisting of the salt of lithium bis(trifluoromethane)sulfonimide ( $\mathrm{Li}^{+} \mathrm{TFSl}$ ) in the solvent of propylene carbonate (PC).

We have presented the operating principle of the tri-layer conducting polymer actuators in detail in our previous papers $[12,13]$. To summarise, when a potential difference is applied between the two conducting polymer layers (PPy) of the actuator, one conducting polymer layer is oxidized while the other is reduced. The oxidized layer takes in anions and expands, while the reduced layer contracts due to the anions leaving this layer. As the whole structure is like a one-end fixed the other end free cantilever beam, this cantilevered structure will bend towards the negative electrode/cathode, as depicted in Fig. S1. The volume change is primarily due to the movement of the charge balancing anions in and out of the polymer layers, and perhaps some solvent molecules move inside the polymer layers, due to osmotic effects to balance the ionic concentration [17]. The actuator parameters which determine the volume change and the dynamic response of the polymer actuators are the thickness, width and length of the active polymer layers and the separator, the type of the salt, ionic concentration of the salt, solvent, and the magnitude and frequency of the potential difference applied $[12,18]$.

\section{Interface design}

In order to achieve good electrical connection to the CP (micro-)actuators that also allows for individual control, we need to develop and an interface. The interface should fulfil certain important design criteria. Firstly, the interface should result in a fine electrical contact minimizing shortcircuiting. It should be able to comprise an electrical circuit to allow for individual control and future electrical components. Preferably it should be easy to make, as well as light-weight in order to make untethered control a possibility.

Flexible Printed Circuit Board (FPCB) fulfils these criteria and is commercially used in a wide range of electronic applications. Therefore we developed a FPCB based interface. The FPCB interface comprised the contacts to the actuators, circuitry to address the individual actuators, and contact pads on to which fine wires will be soldered to connect the interface and thus the actuators to the 
external control electronics. These additional electrical components will be replaced by an on board controller in a later version. We designed the contacts to the top and the bottom electrodes of the tri-layer actuator to be made in a mirror image on the FPCB and all the contact pads on one side (Fig. S2). The contact pads were 500 by $500 \mu \mathrm{m}^{2}$ and the leads were $300 \mu \mathrm{m}$ wide. By folding the FPCB correctly, the top and bottom contact will automatically align and the actuator unit can be inserted in between (see Fig 4a-d).

\section{Materials and Methods}

\subsection{Materials}

PPy (Sigma-Aldrich) was distilled and stored at $-18{ }^{\circ} \mathrm{C}$ prior to usage. Propylene carbonate (PC) and bis(trifluoromethane)sulfonimide lithium salt (LiTFSI) were acquired from Sigma-Aldrich and used as received. $\mathrm{I}_{2}, \mathrm{KI}, \mathrm{HCl}(37 \%)$ and $\mathrm{H}_{2} \mathrm{O}_{2}(30 \%)$ were acquired from Merck and used as received. Porous poly(vinylidene fluoride) (PVDF) filter membrane films used were from Millipore, Immobilon-P, 0.45 $\mu \mathrm{m}$ pore size, thickness $110 \mu \mathrm{m}$. The flexible printed circuit board (FPCB) used was unpatterned DuPont Pyralux LF copper clad LF9110R (a generous gift from DuPont de Nemours, Luxembourg). Thick film photoresist ma-P1275 and corresponding developer ma-P331 were acquired from Microresist Technology, Berlin, Germany.

\subsection{Actuator fabrication}

Fig. 1 illustrates the fabrication steps. First of a piece of PVDF membrane of approximately $5 \times 5 \mathrm{~cm}^{2}$ was cut from the Millipore Immobilon-P roll as received. On to the PVDF membrane a 20-30 nm layer of Au was sputtered on both sides of the PVDF membrane using a Vacutec PlasmaSystems sputter at a pressure of $4.6 \mathrm{mTorr}, 36.6 \mathrm{~cm}^{3} / \mathrm{min}$ Ar flow and a sputter rate of $10 \mathrm{~nm} / \mathrm{min}$. Next, the Au coated PVDF was covered with ma-P1275 thick film photoresist at a thickness of $20 \mu \mathrm{m}$ on one side using a Polos spin coater, followed by a 45 mins post-exposure bake at $100^{\circ} \mathrm{C}$ and a 5 mins exposure using a Karl Suss MJB-3 with a printed photomask from Acreo AB, Norrköping, Sweden, and 5 mins development in ma-P331. The patterning process was repeated on the other side. Thereafter the Au pattern, i.e. the actuator pattern, leads and contact pads, were wet chemically etched for $30 \mathrm{~s}$ using a $\mathrm{KI} / \mathrm{I}_{2}$ solution ( $4 \mathrm{~g} \mathrm{Kl}, 2 \mathrm{~g} \mathrm{I}_{2}$ in $100 \mathrm{ml} \mathrm{H}{ }_{2} \mathrm{O}$ ). The actuator unit is positioned in a conducting frame (etched in the same Au layer) to ensure good electrical contact to all individual actuators during PPy synthesis. Finally the photoresist was stripped using acetone.

Alternatively, a lift-off process to pattern the Au was developed. First, the ma-P1725 photoresist was patterned on both sides of the plain PVDF membrane (approximately $5 \times 5 \mathrm{~cm}^{2}$ ) using the aforementioned process. Thereafter, the 20-30 nm Au layer was sputtered on both sides sequentially using the same parameters and finally patterned using lift-off in acetone.

After the patterning, PPy was electrosynthesized on the patterned Au on both sides simultaneously at a constant current of $0.1 \mathrm{~mA} / \mathrm{cm}^{2}$ for $12 \mathrm{~h}$ in a solution of $0.1 \mathrm{M}$ LiTFSi in PC with $1 \% \mathrm{DI}$ water using an Ivium compactstat or IviumStat (Eindhoven, The Netherlands). In the previous tri-layer actuators $[13,19], \mathrm{PPy}(\mathrm{TFSI})$ was synthesised at $-33^{\circ} \mathrm{C}$, since the properties of PPy are improved by synthesis in organic solvents at low temperatures [20]. However, due to the absence of a low temperature freezer PPy was synthesized at either $-18{ }^{\circ} \mathrm{C}$ or room temperature. A two electrode 
set-up was used for the electrosynthesis: both patterned Au sides were connected parallel to the working electrode lead of the potentiostat and a stainless steel mesh was used as the counter electrode. The reference electrode lead was connected to the counter electrode lead too. After synthesis, the samples were manually cut out in the desired final shape.

\subsection{Interface fabrication}

A piece of approximately $4 \times 4 \mathrm{~cm}^{2}$ was cut out of a sheet of DuPont Pyralux LF9110R FPCB, a singlesided copper-clad Kapton (polyimide) substrate $25 \mu \mathrm{m}$ thick with a $25 \mu \mathrm{m}$ acrylic adhesive. The sample was covered with Shipley S1818 photoresist, $1.5 \mu \mathrm{m}$ thick and exposed using standard photolithography (MJB-3 mask aligner) with a printed photomask. After development, the circuitry (contact pads and lines) was wet chemical etched in the $\mathrm{Cu}$ clad using a $37 \% \mathrm{HCl}$ and $30 \% \mathrm{H}_{2} \mathrm{O}_{2}$ solution (2:1 volume ratio) for $1 \mathrm{~min}$. Thereafter, $0.15 \mathrm{~mm}$ diameter tinned copper fine wires were soldered to the contact pads using a very fine tip soldering iron.

\subsection{Measurements}

The actuators were electrically addressed using an Ivium compactstat or IviumStat (Eindhoven, The Netherlands) with the associated software that also recorded the potentials and currents. For the individual control experiments the Ivium uMUX multiplexer unit was coupled to the potentiostat. Deflection of the actuators was recorded using a Veho VMS-004 Deluxe USB Microscope operating at 30 frames/s and $640 \times 480$ pixels resolution. Deflection was measured using frame grabs of the ultimate positions.

\section{Results}

\subsection{Actuator fabrication}

Devices having 2 or 3 individually controllable PPy tri-layer actuators were microfabricated using either wet chemical etching of Au or lift-off. Fig. 2 shows a "hand" with 3 individually addressable "fingers" all monolithically integrated in a single piece of PVDF to be inserted into the interface as a single unit. The PPy fingers of the hand were $2 \mathrm{~mm}$ wide and $10 \mathrm{~mm}$ long of which $2 \mathrm{~mm}$ is positioned on the PVDF base for the electrical interface. In addition, we fabricated a "finger" having 2 individually addressable joints that were each $5 \mathrm{~mm}$ by $10 \mathrm{~mm}$ and also could be individually addressed (data not shown).

In order to investigate the effect of the microfabrication processing on the performance, a single microfabricated actuator was compared with an unpatterned tri-layer actuator, i.e. manufactured as a large sheet and after synthesis cut out by hand in the same shape as the microfabricated actuators. We could not notice any difference in device appearance, such as the PPy layer, between the unpatterned tri-layer actuator and microfabricated devices. Fig. 3 shows the current response of the two samples. As can be seen, there is almost no difference between the normal and microfabricated actuator indicating that the microfabrication process has no adverse effect on the device functioning. The displacement of the two samples was also identical (data not shown).

\subsection{Interfacing}

Next, we fabricated the FPCB interface. The Cu pattern was etched well on the polyimide foil. Six tinned copper fine wires with a $0.15 \mathrm{~mm}$ diameter to connect the actuator unit to the potentiostat to 
allow for individual control were soldered to the contact pads on the patterned FPCB interface. The interface was then folded. The microfabricated and thereafter cut-out actuator unit was placed in the folded FPCB interface and the assembled unit was pressed together to obtain a good electrical contact between the Cu lines and PPy actuators (see Fig. 4). Due to the flexibility of the FPCB fold, an external force (two alligator clips as can be seen in Fig. 6) was needed to provide good mechanical and thereby good electrical contact. The resistance from solder wire to contact of actuator was 0.4 to $0.6 \mathrm{Ohm}$, of which 0.1 to $0.2 \mathrm{Ohm}$ was the FPCB resistance from contact pads to the actuator contacts depending on length of lines as shown in Fig. S2. The total contact resistance was $62 \mathrm{Ohm}$. When considering the FPCB design, it showed that the PPy actuator to FPCB Cu-contact is the dominating resistance and that the FPCB circuitry resistance is negligible. Also we did not find any change in the resistance of the etched tracks when bending the FPCB substrate.

We compared the influence of the interface on the performance of the actuator. An actuator was first clamped and contacted using a Kelvin clip and thereafter mounted in the FPCB interface. Care was taken to make sure that the length of the actuator (measured from clamp) was the same in both cases. Fig. 5 shows both the current and tip displacement of both interfacing methods. Surprisingly the FPCB interfacing resulted in a higher current and larger displacement of the actuator tip.

Finally, the individual control of the three "fingers" of the "hand" was assessed. All six wires (one pair per PPy actuator) were connected to the UMUX unit of the Ivium potentiostat. Each top and bottom contact was connected to a separate channel, allowing individual control of each finger. The uMUX unit addresses each channel sequentially so that a finger could only be addressed one at the time. All fingers were actuated at $\pm 2 \mathrm{~V}$, but with different pulse periods. The first finger was actuated with $1 \mathrm{~s}$ pulses for 5 cycles, directly thereafter the second at $2 \mathrm{~s}$ pulses for 5 cycles and finally the third at $5 \mathrm{~s}$ pulses for 5 cycles. Fig. 6 shows frame grabs of the actuation sequence. The actuation range was not optimized in this experiment, amongst others PPy was synthesized at room temperature instead of $18 \mathrm{C}$, so the deflection was only a few $\mathrm{mm}$. As can be seen, each finger could be individually addressed, demonstrating the successful microfabrication and interface connection to the individual PPy actuators.

\section{Discussion and conclusions}

We have developed a microfabrication method based on standard photolithography that allows easy fabrication of the patterned PPy tri-layer actuators that can be operated in air. This allowed fabricating more complex structures with individually controllable actuators as demonstrated by a finger with 2 joints and a 3-finger hand and thus for new applications. The fabrication method can be scaled up to allow for batch fabrication of the actuator devices.

Also, we have demonstrated a novel contact method for $\mathrm{CP}$ actuators and the possibility of individual control using this method. The electrical contacting of e.g. tri-layer PPy actuators has been an issue, especially for the thin film actuators [19], where short circuiting of the actuator due to the force of the contact often was a problem. The new method allows for a finer contact reducing the occurrence of failure by short-circuiting. The performance of the actuators using the new interfacing method actually performed better than using a standard Kelvin clip. Using the FPCB interface, the current was higher over the full pulse, meaning that more charge had been injected. The deflection of $\mathrm{CP}$ 
actuators is proportional to the amount of charge injected [21] and explains the increased tip displacement. The higher current indicates that the contact resistance is reduced in the new interfacing. Indeed, we measured a lower contact resistance with the FPCB of $62 \mathrm{Ohm}$ than without the FPCB of nearly $74 \mathrm{Ohm}$.

Since the FPCB interface can be patterned, each CP electrode can be individually addressed and actuated, resulting in individual control. This has been a cumbersome process previously. The FPCB interface can be manufactured easily and its assembly is uncomplicated. The patterned actuator unit is simply mounted between the folded FPCB, resulting in good alignment as well. An additional advantage is that other electronic components, such as batteries and control electronics also could be integrated on the FPCB. The result could be an untethered functioning of EAP powered devices such as microrobots. The FPCB is lightweight. In the present design, the FPCB weighted $25 \mathrm{mg}$ while the actuator unit weighted $38 \mathrm{mg}$. The weight ratio is of course highly dependent on the design and presence of other components, like solder.

A problem with the current FPCB was that the fold was too flexible and did not form a close contact to the actuator. Consequently, an external force was needed to squeeze the two halves together and guarantee a good electrical contact. We did try to use glue to keep the two sides together but did not succeed. The glue did not properly stick the two sides together and decreased the contact resistance. We are currently investigating improving this aspect of the interface including the electrical bonding. This new interfacing method may also be applied to other electroactive polymer devices, such as ion polymer metal composites (IPMC) and dielectric elastomers (DE).

Now that we have established a photolithography based microfabrication process and developed an interface adapted for EAPs, two major hurdles in further development of this technology, we will continue to downscale the actuators to the microdomain as well as develop more complex systems comprising a number of individually controllable PPy microactuators. We are aiming to develop devices down to a few millimetres in size comprising individually controllable actuators of a few hundred micrometres in size.

\section{Acknowledgement}

The authors wish to thank Dr Wen Zheng, Dr Alexey Pan, Dr Olga Shcherbakova at UoW and Dr Chunxia Du at LiU for their assistance. Funding has been supplied by the European Science Foundation COST Action MP1003 ESNAM (European Scientific Network for Artificial Muscles) and COST-STSM-MP1003-8971, the Swedish Research Council (VR - 2010-6672), the Knut \& Alice Wallenberg Stiftelse (LiU-2010-00318), Linköping University, the EU Erasmus program, and the Australian Research Council for partial financial assistance through the Centers of Excellence, Discovery Projects (DP0878931) and Fellowships.

\section{References}

[1] E.W.H. Jager, E. Smela, O. Inganäs, Microfabricating Conjugated Polymer Actuators, Science, 290(2000) 1540-5.

[2] Q. Pei, O. Inganäs, Conjugated polymers and the bending cantilever method: electrical muscles and smart devices, Adv Mater, 4(1992) 277-8. 
[3] R.H. Baughman, L.W. Shacklette, R.L. Elsenbaumer, E.J. Plichta, C. Becht, Micro electromechanical actuators based on conducting polymers, in: P.I. Lazarev (Ed.) Molecular Electronics, Kluwer Academic Publishers, Dordrecht, 1991, pp. 267-89.

[4] T.F. Otero, J.M. Sansinena, Artificial muscles based on conducting polymers, Bioelectrochemistry and Bioenergetics, 38(1995) 411-4.

[5] M.R. Gandhi, P. Murray, G.M. Spinks, G.G. Wallace, Mechanism of electromechanical actuation in polypyrrole, Synth Met, 73(1995) 247-56.

[6] E. Smela, O. Inganäs, I. Lundström, Controlled folding of micrometer-size structures, Science, 268(1995) 1735-8.

[7] K. Svennersten, M. Berggren, A. Richter-Dahlfors, E.W.H. Jager, Mechanical stimulation of epithelial cells using polypyrrole microactuators, Lab on a Chip, 11(2011) 3287 - 93.

[8] Y. Berdichevsky, Y.-H. Lo, Polymer Microvalve Based on Anisotropic Expansion of Polypyrrole, Mat Res Soc Symp Proc, Materials Research Society2004, pp. A4..1-7.

[9] E. Smela, M. Kallenbach, J. Holdenried, Electrochemically driven polypyrrole bilayers for moving and positioning bulk micromachined silicon plates, J Microelectromech Syst, 8(1999) 373-83.

[10] E.W.H. Jager, O. Inganäs, I. Lundström, Microrobots for Micrometer-Size Objects in Aqueous Media: Potential Tools for Single Cell Manipulation, Science, 288(2000) 2335-8.

[11] F. Vidal, J.F. Popp, C. Plesse, C. Chevrot, D. Teyssie, Feasibility of conducting semiinterpenetrating networks based on a poly(ethylene oxide) network and poly(3,4ethylenedioxythiophene) in actuator design, Journal of Applied Polymer Science, 90(2003) 3569-77.

[12] Y. Wu, G. Alici, G.M. Spinks, G.G. Wallace, Fast trilayer polypyrrole bending actuators for high speed applications, Synth Met, 156(2006) 1017-22.

[13] G. Alici, V. Devaud, P. Renaud, G. Spinks, Conducting polymer microactuators operating in air, J Micromech Microeng, 19(2009) 025017.

[14] A. Khaldi, Conducting interpenetrating polymer network sized to fabricate microactuators, Appl Phys Lett, 98(2011) 164101.

[15] K. Kruusamäe, P. Brunetto, S. Graziani, A. Punning, G. Di Pasquale, A. Aabloo, Self-sensing ionic polymer-metal composite actuating device with patterned surface electrodes, Polymer International, 59(2010) 300-4.

[16] E. Jager, B. Gaihre, G. Alici, G. Spinks, Patterning of polypyrrole trilayer actuators working in air for microrobotics, EuroEAP 20122012.

[17] L. Bay, T. Jacobsen, S. Skaarup, K. West, Mechanism of actuation in conducting polymers: Osmotic expansion, J Phys Chem B, 105(2001) 8492-7.

[18] F. Carpi, R. Kornbluh, P. Sommer-Larsen, G. Alici, Electroactive polymer actuators as artificial muscles: are they ready for bioinspired applications?, Bioinspiration \& Biomimetics, 6(2011) 045006.

[19] B. Gaihre, G. Alici, G.M. Spinks, J.M. Cairney, Synthesis and performance evaluation of thin film PPy-PVDF multilayer

electroactive polymer actuators, Sensors and Actuators A, 165(2011) 321-8.

[20] W. Zheng, J.M. Razal, G.M. Spinks, V.-T. Truong, P.G. Whitten, G.G. Wallace, The Role of Unbound Oligomers in the Nucleation and Growth of Electrodeposited Polypyrrole and Method for Preparing High Strength, High Conductivity Films, Langmuir, 28(2012) 10891-7.

[21] T.F. Otero, J.M. Sansiñena, Bilayer Dimensions and Movement in artificial muscles, Bioelectrochemistry and Bioenergetics, 42(1997) 117-22. 


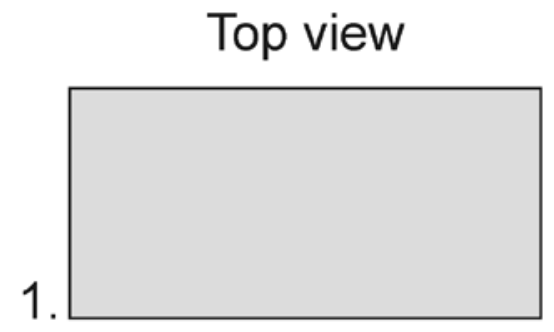

Cross section
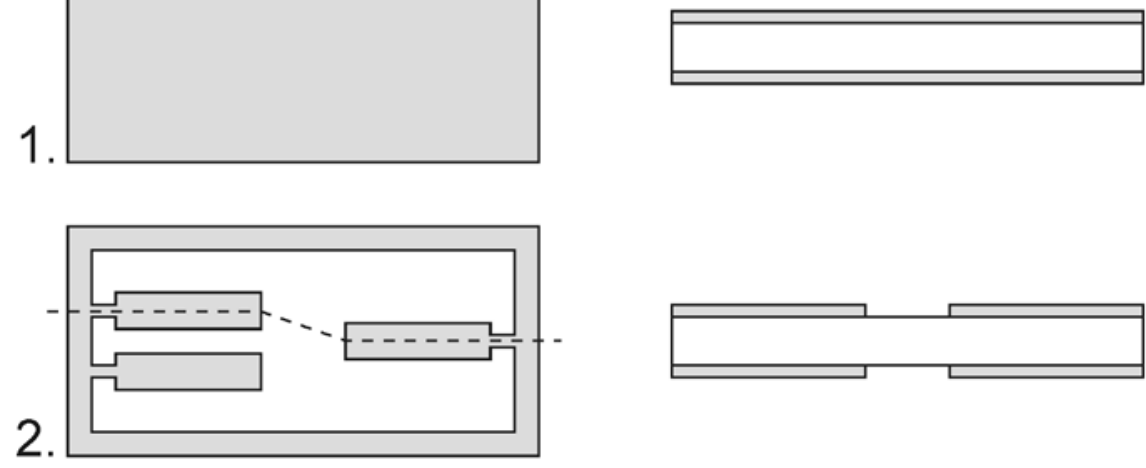

3.
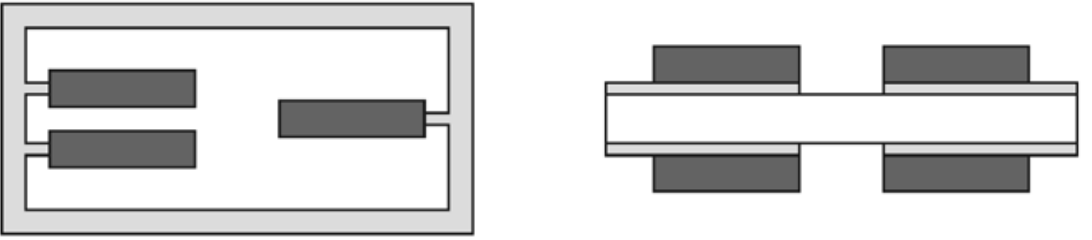

4.
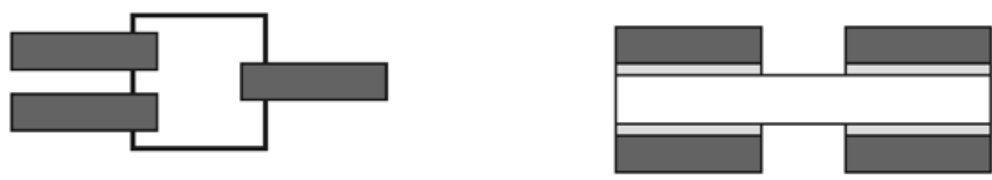

Fig. 1. Illustration of the process steps for microfabricating the actuator unit, top view and cross section along the dashed line. A $5 \times 5 \mathrm{~cm}^{2}$ piece of commercial PVFD membrane is cut from the roll. 1 . Sputter deposition of 20-30 nm Au on both sides of the PVDF. 2. Wet chemical etching of the Au actuator structure and conducting frame. 3. Electrochemical synthesis of PPy(TFSI). 4. The final actuator unit is cut out from the membrane and conducting frame. The processing using lift-off is similar except for the Au layer being patterned using lift-off. 


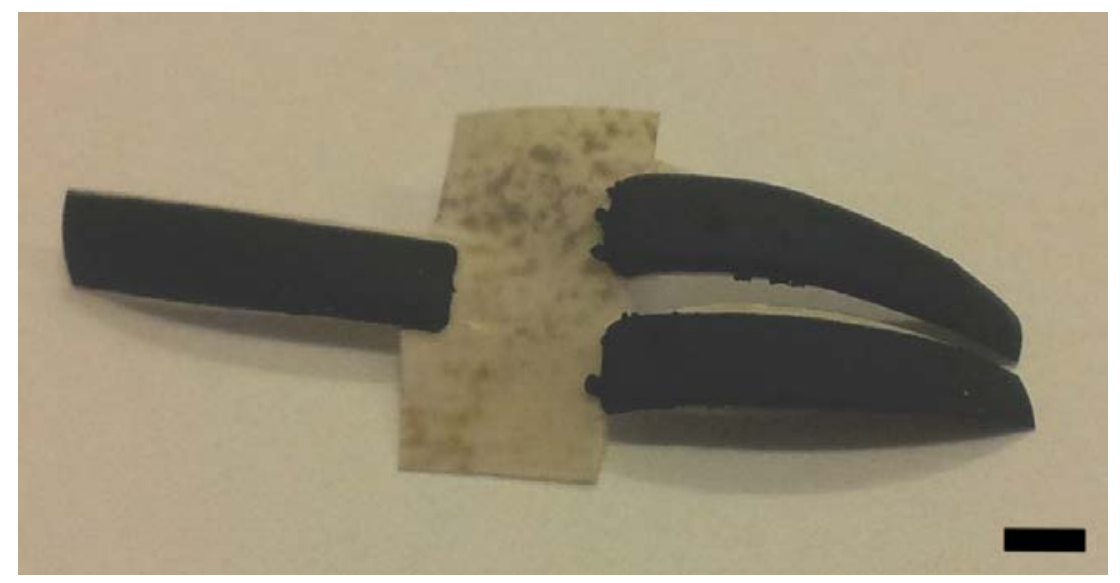

Fig. 2. A photograph of a "hand" with 3 individually addressable "fingers", microfabricated and patterned PPy tri-layer actuators, $10 \mathrm{~mm}$ long and $2 \mathrm{~mm}$ wide. Scale bar $2 \mathrm{~mm}$. 


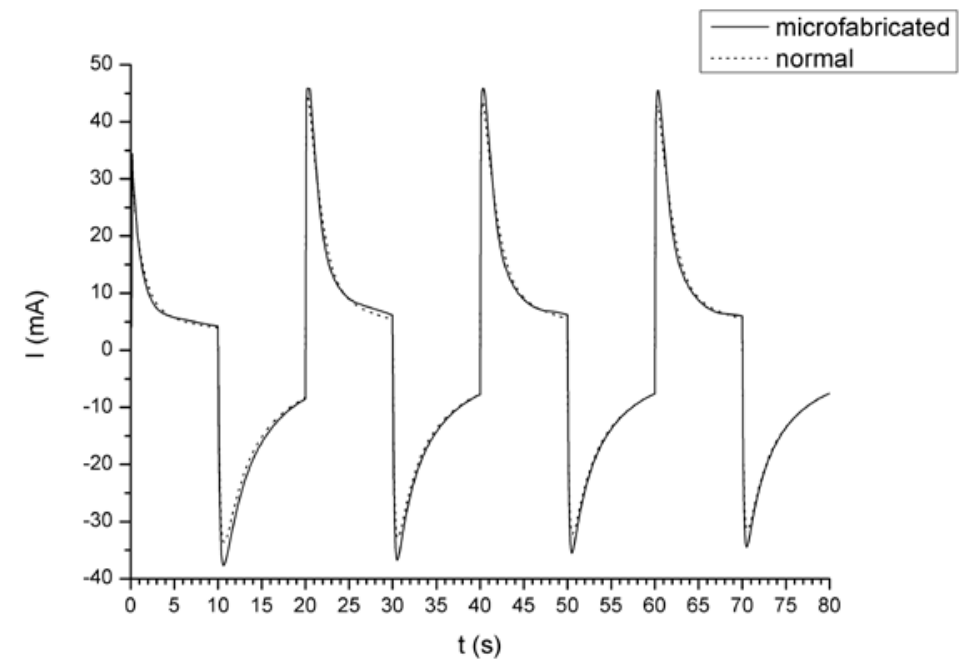

Fig. 3. The current transient of a standard, normally fabricated and a microfabricated actuator trilayer PPy actuator under $V_{\text {applied }}=1.5 \mathrm{~V}$. 
a.
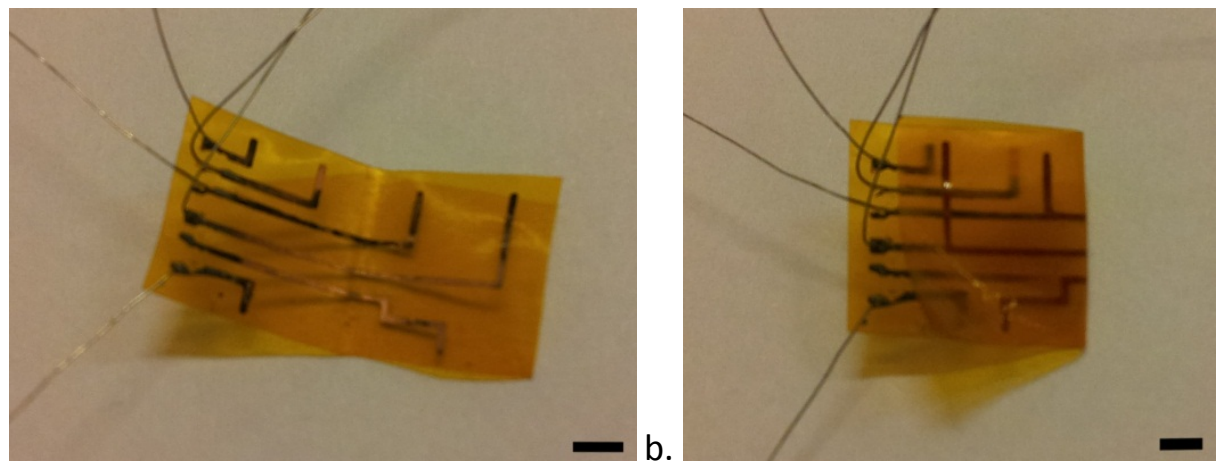

c.
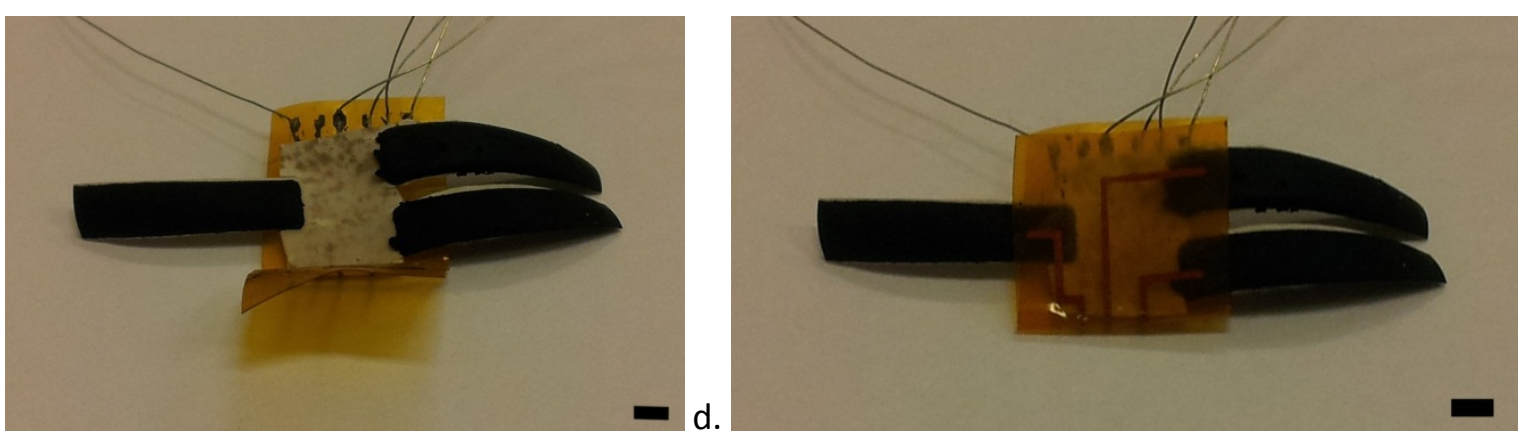

Fig. 4. Assembly of the actuator device. The FPCB interface in the open (a) and closed (b) position. The actuator unit is placed in the open FPCB interface with the actuators aligned to the Cu leads (c). The FPCB interface is clamped around the actuator unit (d). Scale bars $2 \mathrm{~mm}$. 

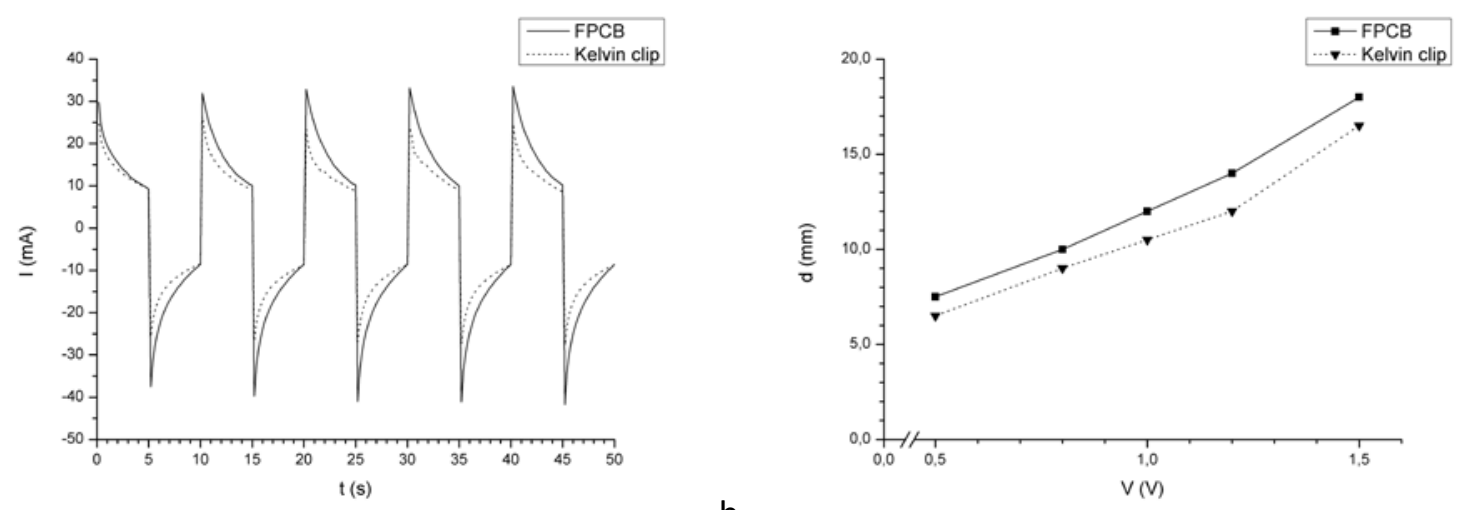
a.

b

Fig. 5. The current (a) and displacement (b) of a PPy tri-layer actuator $(2 \mathrm{~mm} \times 9 \mathrm{~mm})$ contacted by a Kelvin clip or the FPCB interface. 

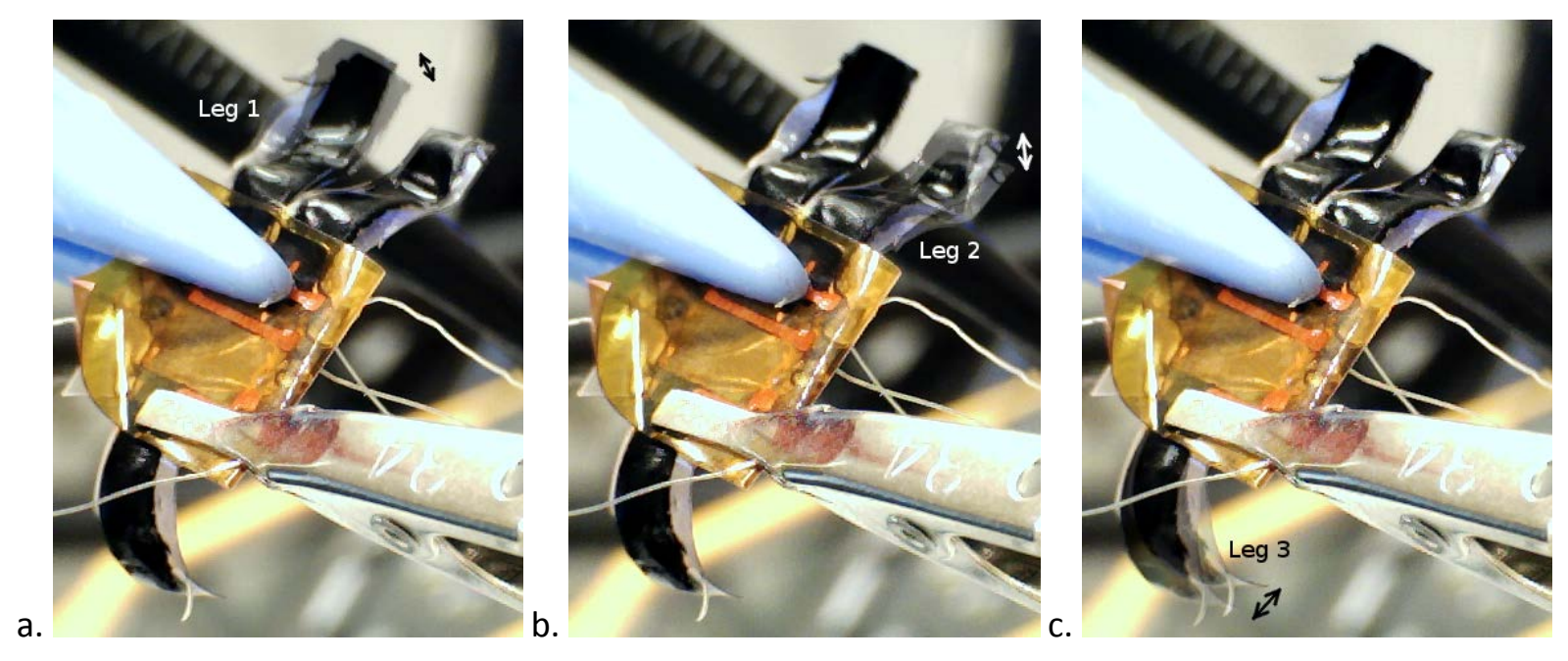

Fig. 6. Overlay images of two frame grabs showing individual actuation of each "leg". In each image only the addressed leg moves, while other legs do not. The actuator unit was not optimized so the deflection was only a few $\mathrm{mm}$. The full movie can be downloaded at..... 


\section{Supplementary material}<smiles></smiles>
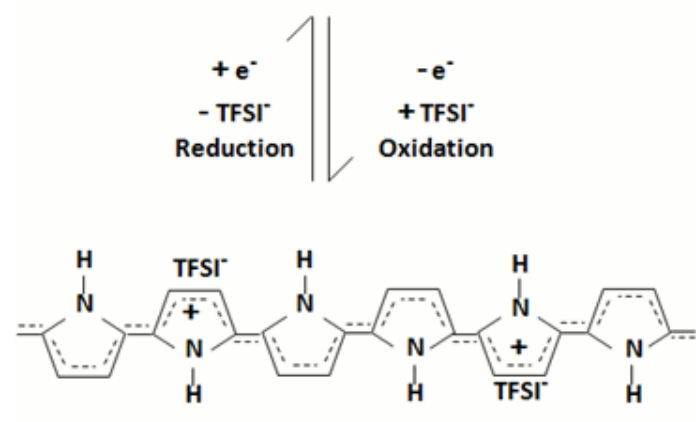

b.

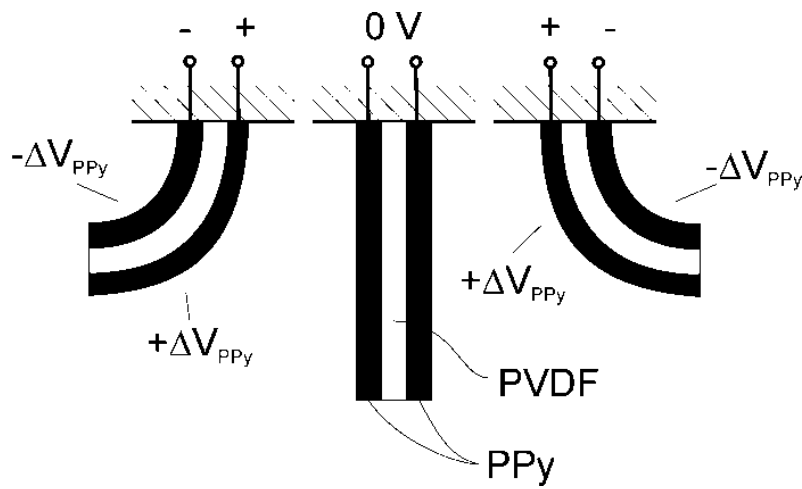

Fig. S1. (a) Illustration to of the electrochemical oxidation and reduction of PPy doped with TFSI anions. (b) Schematic representation of the bending principle of the tri-layer PPy actuator. The trilayer actuator consists of a PVDF membrane (white) with a thin layer of sputtered Au (not shown) coated with TFSI doped PPy (black) on both sides of the membrane. At the positively charged electrode PPy is oxidized and anions from the electrolyte contained inside the pores of the PVDF membrane move into the PPy causing a volume expansion. At the negative electrode, the PPy is reduced causing expulsion of anions and thus volume shrinkage. This process is fully reversible causing a rocking motion.

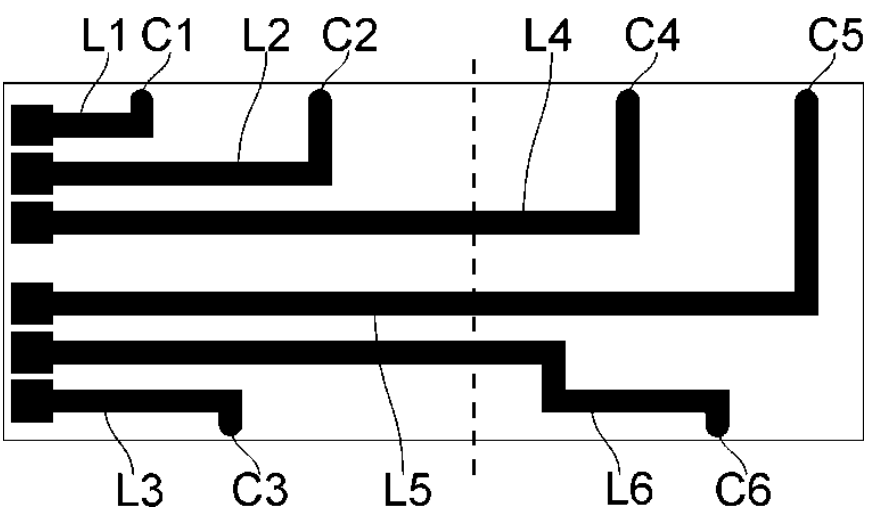

Fig. S2. The layout of the FPCB interface. The bottom (C1 - C3) and the top (C4-C6) contacts to the tri-layer actuator were made to be in a mirror image on the FPCB. All the contact pads were positioned on one side (left in this figure) and were 500 by $500 \mu \mathrm{m}^{2}$. The leads were $300 \mu \mathrm{m}$ wide and the lengths were $L 1=2.61 \mathrm{~mm} ; \mathrm{L} 2=7.59 \mathrm{~mm} ; \mathrm{L} 3=4.14 \mathrm{~mm} ; \mathrm{L} 4=14.57 \mathrm{~mm} ; \mathrm{L} 5=15.8 \mathrm{~mm} ; \mathrm{L} 6=21.01$ $\mathrm{mm}$, respectively. By folding the FPCB along the dashed line, the top and bottom contacts will automatically align and the actuator unit can be inserted in between (see Fig $5 a-d$ ). 


\section{Biographies}

Edwin Jager is Associate Professor in the Biosensors and Bioelectronics Centre at the Dept. of Physics, Chemistry and Biology (IFM), Linköping University, Sweden, which he joined in 2011. He received his M.Sc.Eng. degree (ir.) in Applied Physics at University of Twente, The Netherlands in 1996 and his PhD in Applied Physics at Linköping University, Sweden in 2001, where he worked on conducting polymer microactuators for biomedicine and cell biology. From 2000 to 2007 he worked as Chief Technology Officer and co-founder at the spin-off company Micromuscle $A B$, commercializing medical applications of the polymer actuator technology. Thereafter, he returned to academia as Assistant Professor in the Organic Electronics group at the Dept of Science and Technology at the Norrköping campus until spring 2011. His research interests include conducting polymers, bioelectronics and polymer (micro-)actuators for cell biology, medicine and robotics.

Nirul Masurkar holds a MSc in Electronics Circuit Design and Manufacturing from the University of Dundee, UK, in 2012 and a Bachelor's of Engineering degree in Electrical (Electronics and Power) from St. Vincent Pallotti college of Engineering and Technology, Nagpur in 2009. He is completed his master thesis in Linkoping University, Sweden. He was a research student in Organic Electronics Productronica, Munich, Germany. He is currently a junior researcher at Jawaharlal Nehru Centre for Advanced Scientific Research, India.

Nnamdi Felix Nworah, received a M.Sc in Biomedical Engineering from Linköping University, Sweden in 2012. Obtained his B.Tech in Physics Electronics from Nnamdi Azikiwe University Awka, Nigeria in 2007. He has worked in area of characterization and fabrication of polypyrrole microactuators at Linköping University Sweden

Babita Gaihre completed her master degree in Organic Chemistry (2001) from Tribhuvan University, Nepal and PhD (2008) from Chonbuk National University, South Korea. She was appointed as Associate Research Fellow at the University of Wollongong in 2009. Her PhD work involved synthesis and characterization of surface modified nanoparticles and study of potential application of these surface modified nanoparticles as a carrier system for targeted delivery of drug. Her initial research in the Department of Engineering, University of Wollongong involved fabrication and performance study of microactuators made up of conducting polymers. After successful completion of her tenure in the department she worked as a research fellow in the Intelligent Polymer Research Institute (IPRI). Her current research involves patterning of conducting polymer for the fabrication of electrochromic and bionic device.

Gursel Alici received a PhD degree in Robotics from the Department of Engineering Science, Oxford University, UK, in 1994. He is currently a Professor at the University of Wollongong, NSW, Australia, where he is the head of School of Mechanical, Materials and Mechatronic Engineering. His current research interests include intelligent mechatronic systems involving mechanisms/serial/parallel robot manipulators, system dynamics and control, micro/nano robotic systems for medical applications, and modeling, analysis, characterization, control of conducting polymers as macro/micro/nano sized actuators and sensors for robotic and bio-inspired applications. He was a technical editor of 
IEEE/ASME Transactions on Mechatronics from 2008 to 2012, and is a member of the Mechatronics National Panel formed by the Institution of Engineers, Australia. He has produced over 200 refereed publications in his areas of research and is also the recipient of the Outstanding Contributions to Teaching and Learning (OCTAL) award from the University of Wollongong in 2010.

Geoffrey Spinks received his PhD from the University of Melbourne in 1990 for his work on the fracture behaviour of unsaturated polyesters. Appointed as lecturer at the University of Wollongong in 1990, he has continued a research interest in the mechanical behaviour of polymers. Most of his research has been in the area of mechanical actuators, including conducting polymers, hydrogels, carbon nanotubes and their composites. He has published over 150 journal papers relating to new materials development and characterisation, actuation mechanisms and applications. Professor Spinks is currently an Australian Research Council Professorial Fellow. 